ARTICLE OPEN

\title{
New classification of periodontal diseases (NCPD): an application in a sub-Saharan country
}

William Ndjidda Bakari ${ }^{1}{ }^{1}$, Diabel Thiam ${ }^{1}$, Ndeye Lira Mbow ${ }^{1}$, Anna Samb ${ }^{1}$, Mouhamadou Lamine Guirassy ${ }^{1}$, Ahmad Moustapha Diallo (iD) ${ }^{1}$, Abdoulaye Diouf ${ }^{1}$, Adam Seck Diallo ${ }^{1}$ and Henri Michel Benoist ${ }^{1}$

PURPOSE: To determine the clinical and radiological profile of periodontitis according to the 2018 NCPD, in a Dakar (Senegal) based periodontal clinic.

METHODS: This is a descriptive study based on patient's records in the periodontology clinic. The study was conducted between November 2018 and February 2020 (15 months). All periodontitis cases were included in the study. Incomplete records (due to lack of radiographic workup or unusable periodontal charting) were excluded. Periodontitis diagnosis was established based on criteria used in the 2018 NCPD. Statistical analysis was carried out using SPSS version 20.0, with the significance threshold set at 0.05 . RESULTS: A total number of 517 patient records were collected during the study period but only 127 periodontitis records were complete. The mean age of participants was $46.8 \pm 13.8$ years and $63.8 \%$ of participants were males. The mean plaque index and bleeding on probing (BOP) were $74 \% \pm 21.3$ and $58.1 \% \pm 25.1$, respectively. The mean maximum clinical attachment loss was $8.7 \mathrm{~mm} \pm 2.7$, with a probing depth greater than $6 \mathrm{~mm}$ present in $50.4 \%$ of the sample. The median number of missing teeth was 3 (interquartile range 5-1). Pathological mobility was present in $60.6 \%$ of the patients and $78.0 \%$ had occlusion problems. Bone crest defect at the most affected site was moderate in $52.8 \%$ of cases. The ratio of bone loss to age greater than one concerned $66.1 \%$ of the sample. Generalised (81.9\%), Stage IV (70.1\%) and grade C (69.3\%) were the most encountered diagnosis. The disease severity was associated with age $(r=0.241 ; P<0.001)$, BOP $(r=0.230 ; P=0.013)$ and the number of teeth with pathological mobility $(r=0.318 ; P<0.001)$.

CONCLUSION: Patients with periodontitis in this study had advanced forms of the disease and required multidisciplinary care. Clinical hindsight is necessary to improve this classification.

BDJ Open (2021)7:16

; https://doi.org/10.1038/s41405-021-00071-8

\section{INTRODUCTION}

Periodontitis is a disease-causing the progressive destruction of the tooth-supporting apparatus, characterized by a clinical attachment loss (CAL), a radiographically assessed alveolar bone loss, the presence of periodontal pockets and gingival bleeding. ${ }^{1}$ It is a polymicrobial disease with an inflammatory burden that can ultimately cause tooth loss in the absence of adequate treatment. ${ }^{2}$ Periodontal diseases have been subjected to several classifications over the years, in view to better define and circumscribe the different pathologies contained in it, providing a suitable tool for research, therapeutics and epidemiology.,4 Ever since pyorrhoeaa alveolaris was first classified, many experts consensus meetings were held to continuously upgrade these classifications to render them compatible to the best available knowledge at the time. ${ }^{5}$ Therefore, a consensus conference was held in 2017 between the European (EFP: European Federation of Periodontology) and the American (AAP: American Association of Periodontology) periodontal societies to update the latest Armitage Periodontal Disease Classification of $1999 .^{4}$ This new classification of periodontal diseases (NCPD) including; peri-implant pathologies, proposes a definition of periodontitis and periodontal health. ${ }^{4}$ With the current classification, which actually introduces a new approach for periodontal screening conditions, it seemed interesting to look at the profile of patients with periodontitis in our setting. Thus, the aim of this study is to determine the clinical and radiological profile of periodontitis on a Senegalese population using the 2018 classification in a Dakar based periodontal clinic.

\section{METHODS}

Study design and settings

This was a descriptive study based on the records of patients having consulted at the periodontology clinic of the Institute of Odontology and Stomatology of the Cheikh Anta Diop University of Dakar (IOS/UCAD). Recruitment spread over the period of November 2018 to February 2020 (15 months). The records of patients who had periodontitis were included in the study. All records, whose final diagnosis was not periodontitis (periodontal health, gingivitis, muco-gingival defects, necrotizing periodontal diseases, periodontitis as manifestations of systemic disease) were not included and considered as incomplete records (lack of radiographic workup or unusable periodontal charting) were excluded (Fig. 1). At first, a minimum of 15 teeth (present in the mouth) were required in patients for consideration for a periodontal clinic. Patients were referred or invited by students as part of their periodontal clinic.

\footnotetext{
'Department of Periodontology, Institute of Odontology and Stomatology, CheikhAnta Diop University, Dakar, Senegal
}

Correspondence: William Ndjidda Bakari (bakariwilliam@yahoo.fr)

Received: 12 January 2021 Revised: 30 March 2021 Accepted: 6 April 2021

Published online: 26 April 2021 


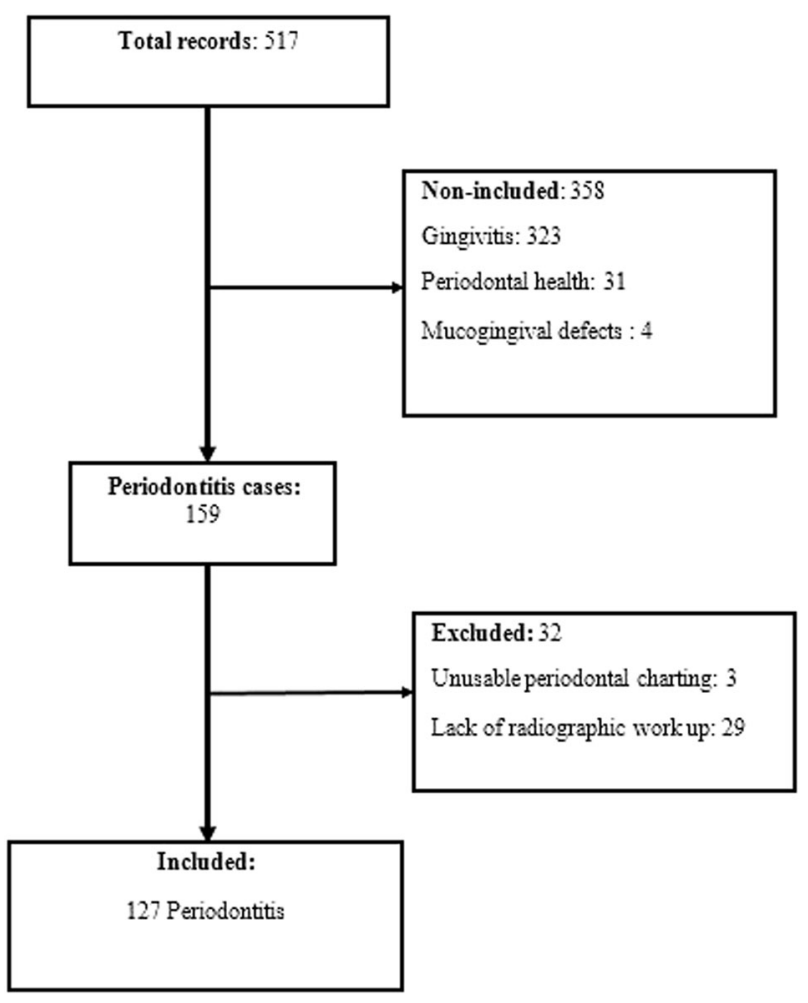

Fig. 1 Flow chart. Showing the number of periodontitis cases during the selection process.

Data collection and diagnostic criteria

The diagnosis for periodontitis in this study was established on clinical and radiological criteria used in the 2018 NCPD. ${ }^{6}$ The data collected were mostly on: sociodemographic variables (sex, age), risk factors (current smoking, and glycated haemoglobin levels); clinical variables including: maximum clinical attachment loss (maxCAL), probing depth (PD), tooth mobility $>2$ (Mühlemann et $\left.a .^{7}\right)$, plaque index (PI) (O'Leary et al. $\left.^{8}\right)$, bleeding on probing (BOP) (Ainamo and Bay ${ }^{9}$ ), number of missing teeth and furcation involvement (Hamp et al. $^{10}$ ). Radiographic variables considered were the ratio of bone loss percentage to age, phenotype, bone resorption (horizontal, vertical, or mixed), the general level of bone destruction, the extension of alveolysis (localised $<30 \%$ of teeth; generalised $>30 \%$ of teeth) and bone ridge defect (moderate and severe). The Williams periodontal probe (Michigan $\mathrm{O}$ probe, $\mathrm{Hu}-$ Friedy Mfg. Co, Chicago, IL USA) was used for periodontal probing. Measurements of CAL and probing pocket depth were made at 6 (six) sites on each tooth according to the charting form used in the IOS/UCAD periodontology department. Wisdom teeth were included in the count for missing teeth. CAL and PD were estimated by the distances ranging from the cement-enamel junction (CEJ) and the free edge of the gingiva to the bottom of the sulcus. Assessment of alveolar bone lysis was made proximal to the most affected site using the technique proposed by Kornman and Papapanou ${ }^{11}$. Bite collapses, drifting and flaring were considered occlusion disorders. The radiographic assessment included an orthopantomogram and retro-alveolar radiographs (performed with the same apparatus for all patients) for the most affected sites identified on the orthopantomogram. Bone loss was measured by estimating the percentage distance between CEJ to the deepest part of the bone defect along the root length. Under $\mathrm{X} 2$ magnification with the light of the negatoscope (Fazzini negatoscope SS. Padanasuperiore, 317.20090 Vimodrome, Mi Italy), the root was divided into three parts from the CEJ to the root apex. The first part represented the coronal third, the second part the middle third and the third part the apical third. ${ }^{11}$
Interdental bone loss extending from the coronal third to the median third (33-50\%) was considered as a moderate ridge defect. While a bone loss, exceeding $50 \%$ (from the middle third to the apical third) was regarded as a severe ridge defect. The general bone resorption level was classified as mild (coronal third), moderate (middle third) and terminal (apical third). The phenotype was assessed by seeking the proportionality between the PI found and the bone resorption observed at the most affected radiological site. There were therefore three types of phenotype: heavy biofilm deposits for a low level of bone destruction, destruction proportional to the biofilm, and destruction exceeding biofilm deposits.

The final diagnosis was made based on criteria of severity and complexity for the stage and according to the ratio of bone loss to age and risk factors for the grade (Tables 1 and 2). Two independent reviewers made the diagnosis and differences were resolved by consensus on the intervention of a third reviewer. This NCPD was the subject of several training sessions in the department to calibrate the examiners.

Statistical analysis

Statistical analysis was carried out using SPSS (Statistical Package for Social Sciences) version 20.0. Qualitative variables were expressed as frequencies, percentages, and quantitative variables as means and standard deviations. Linear regression was used to study the relationship between the severity of the impairment designated by the maxCAL and age, $\mathrm{PI}, \mathrm{BOP}$, number of mobile teeth, and number of missing teeth. The significance threshold was set at 0.05 .

\section{RESULTS}

A total number of 517 patient records were collected during the study period. Periodontitis was diagnosed in 159 patients, giving a prevalence of $30.8 \%$. Only 127 periodontitis records were complete and had a radiographic record available.

The population was predominantly male with 81 (63.8\%) individuals (Table 3 ). The mean age was $46.8 \pm 13.8$ years with a range of 21-74 years. The age distribution revealed a peak in the population after 20 and 50 years old (Fig. 2). The majority of those working had jobs in the informal sector (48.0\%) and $23.6 \%$ presented a periodontal risk factor of smoking and/or diabetes (Table 3).

According to clinical data (Table 4), the mean PI and BOP were $74 \% \pm 21.3$ and $58.1 \% \pm 25.1$, respectively. The mean maxCAL was $8.7 \mathrm{~mm} \pm 2.7$ with a range of $2-16 \mathrm{~mm}$. PD greater than $6 \mathrm{~mm}$ $(P D \geq 6 \mathrm{~mm})$ was present in $50.4 \%$ of the sample. The median number of missing teeth was 3 for a mean of 5 mobile teeth. Pathological mobility was present in $60.6 \%$ of the patients and 78.0\% had occlusion problems (Fig. 3 ).

Concerning the radiological features (Table 5 ), alveolysis was generalised $(81.9 \%)$, mixed $(66.1 \%)$ and moderate $(54.3 \%)$. The ridge defect at the most affected site was moderate in $52.8 \%$ of cases. The ratio of bone loss to age greater than one (BL/age $>1$ ) concerned $66.1 \%$ of the sample. Moreover, the phenotype was proportional in most cases (61.4\%). There was a discrepancy between phenotype and age-related bone loss ratio. For a proportional phenotype, the ratio of $\mathrm{BL} / \mathrm{age}$ was mostly greater than one (Fig. 4).

Stage IV (70.1\%) and grade C (69.3\%) were the most encountered diagnosis. The extension was generalised in $81.9 \%$ of the sample (Table 6). Thus, the most frequently mentioned clinical form was generalised stage IV grade $C$ periodontitis; found in $50.4 \%$ of the sample (Fig. 5). The disease severity was associated with age $(r=0.241 ; P<0.001)$, BOP $(r=0.230 ; P=0.013)$ and the number of teeth with pathological mobility $(r=0.318 ; P<0.001)$ (Table 7). The maxCAL thus increased significantly with age, BOP and the number of mobile teeth. 

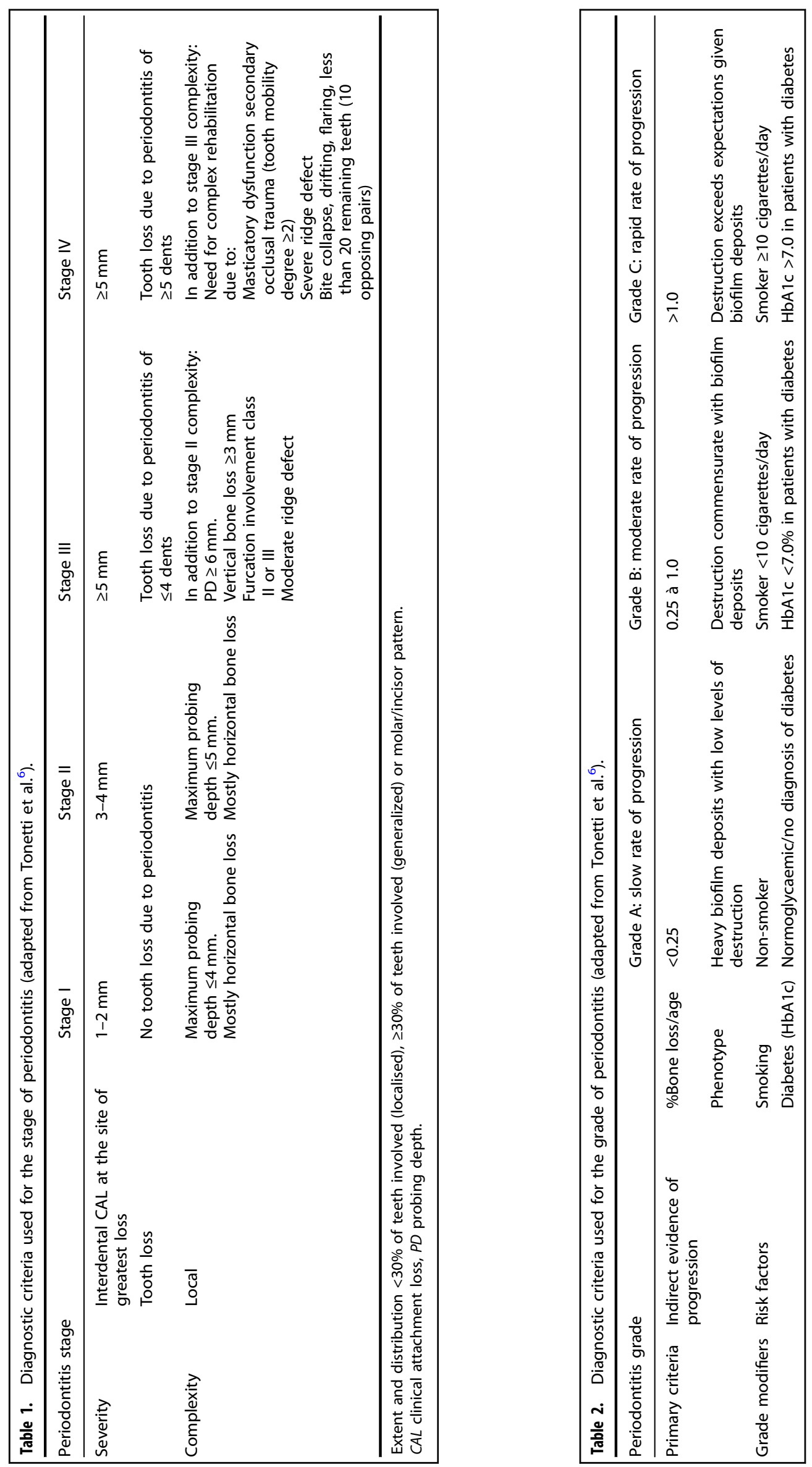
Table 3. Socio-demographic characteristics.

\begin{tabular}{llr}
\hline Variables & $N=127$ & $\%$ \\
\hline Sex & & \\
$\quad$ Female & 46 & 36.2 \\
Male & 81 & 63.8 \\
Age (years) & Mean: $46.8 \pm 13.8$ min 21; max 74 & \\
Occupation & & 29.9 \\
Jobless & 38 & 48.0 \\
Informal & 61 & 14.2 \\
Private & 18 & 7.9 \\
Public & 10 & \\
Risk factor & & 76.4 \\
No & 97 & 23.6 \\
Yes & 30 & 7.1 \\
Smoking & & 8.7 \\
Smoker $<10$ cigarettes/day & 9 & 84.3 \\
Smoker $\geq 10$ cigarettes/day & 11 & \\
No smoking & 107 & 10.2 \\
Diabetes HbA1c (\%) & & 2.4 \\
HbA1c $>7$ & 17 & 84.3 \\
HbA1c $<7$ & 3 & \\
No diabetes & 107 &
\end{tabular}

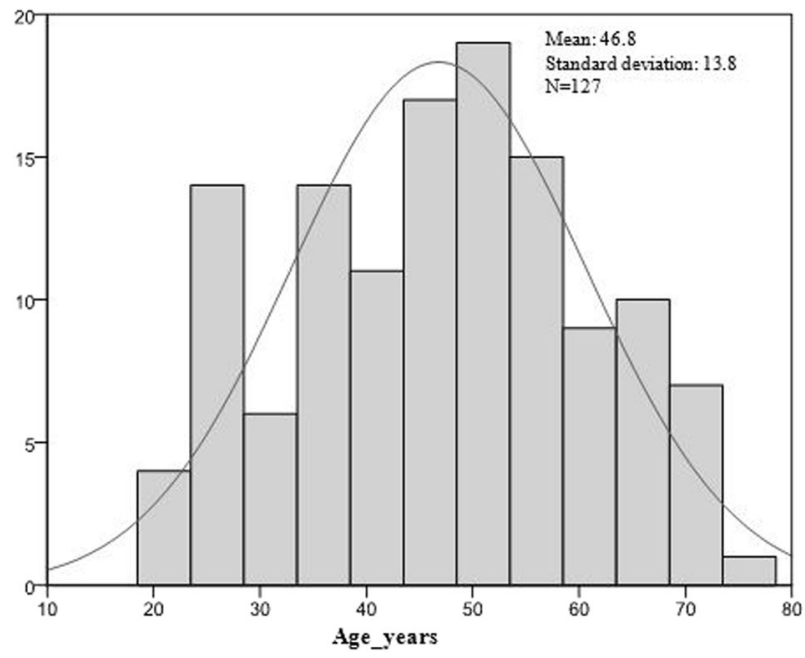

Fig. 2 Age distribution. Showing a peak around the age of 50.

Table 4. Clinical features.

\begin{tabular}{llllll}
\hline Variables & Mean & $\begin{array}{l}\text { Standard } \\
\text { deviation }\end{array}$ & Minimum & Maximum & Median \\
\hline MaxCAL (mm) & 8.7 & 2.7 & 2 & 16 & 8 \\
PI (\%) & 74.7 & 21.3 & 13 & 100 & 78 \\
BOP (\%) & 58.1 & 25.1 & 11 & 100 & 60 \\
Missing teeth & 5 & 4 & 0 & 17 & 3 \\
Tooth mobility $^{a}$ & 4 & 6 & 0 & 31 & 2 \\
\hline
\end{tabular}

MaxCAL maximum interdental clinical attachment loss, PI plaque index $O$ 'Leary, $B O P$ bleeding on probing ainamo \& bay.

a'Degree $\geq 2$

\section{DISCUSSION}

This study aimed to determine the clinical and radiological profile of periodontitis according to the NCPD of 2018. Periodontitis occurs mostly in male patients, with a mean age of 47 years and those working in the informal job sector. Clinically, the patients had a mean maxCAL of $8.7 \mathrm{~mm}$ with $\mathrm{PD} \geq 6 \mathrm{~mm}$, a mean $\mathrm{PI}$ and BOP of $74 \%$ and $58 \%$ respectively, with tooth mobility degree $\geq 2$ (Mühlemman et al. ${ }^{7}$ ), furcation grade II or III (Hamp et al. ${ }^{10}$ ) involvement and occlusion disorders. Radiographically, bone lysis was generalised, mixed with moderate ridge defects. The bone loss/age ratio was $>1$ for a proportional phenotype. Periodontitis was generalized stage IV and grade $C$.

Benoist et al. ${ }^{12}$ reported out of 564 patients with periodontitis, a male predominance of $53 \%$. Whilst Ravida et al. ${ }^{13}$ and Graetz et al. $^{14}$ found a female predominance of $52.1 \%$ and $60.2 \%$, respectively, on 251 and 292 patients received in a university periodontology clinic according to the new classification. Though these studies report a higher prevalence of periodontitis in women, male predominance has been shown in the literature. ${ }^{15}$ However, no clear association was shown to exist between gender and the occurrence of periodontitis. ${ }^{15}$

The mean age was $46.8 \pm 13.8$ years. Benoist et al. ${ }^{12}$ found a mean age of $40.4 \pm 14.9$ years for chronic periodontitis and $28.1 \pm 8.9$ years for aggressive periodontitis. Chronic periodontitis represented $73.2 \%$ of their sample. Ravida et al. ${ }^{13}$ found a mean age of $47.3 \pm 12$ years while Graetz et al. ${ }^{14}$ found a mean age of $45.1 \pm 9.6$ years. These results are close to our findings confirming the trend observed. After the 1999 classification, it was acknowledged that periodontitis can occur at any age and the chronic and aggressive forms of the disease were described. ${ }^{3}$ In this recent classification, age plays a role in the indirect assessment of the disease progression by the bone loss to age ratio but not as a risk factor. Only diabetes and smoking remain considered as grade-modifying risk factors in the diagnosis of periodontitis. ${ }^{1}$ Nevertheless, age was associated with the severity of the disease in this study.

Interdental CAL is the main criterion for assessing the severity of the periodontal disease. ${ }^{11,16}$ The inclusion of CAL at the most affected tooth is an innovation in this classification. ${ }^{6}$ Clinically, our patients had a mean maxCAL of $8.7 \mathrm{~mm}$ indicating the severity of the disease observed. CAL in the study by Benoist et al. ${ }^{12}$ for chronic periodontitis was $4.4 \mathrm{~mm}$ representing a mean value that was not based on the most affected tooth, which explains why it is lower than that found in this study. Graetz et al. ${ }^{14}$ obtained a mean of $7.5 \mathrm{~mm}$. The NCPD considers the maximum of the CAL, therefore values higher than those observed in studies using Armitage's classification makes sense. ${ }^{1}$

Half of our participants had generalised stage IV grade C periodontitis. Ravida et al. ${ }^{13}$ in their study found stage III (50\%) and grade $B(66.1 \%)$ as the majority. Their study was retrospective, and the data recorded for the stage including information on complexity were not complete. Stage III/grade C (55.77\%) was the most common in the study by Graetz et al. ${ }^{14}$ on 251 periodontitis cases. In Graetz's study, risk factors were not documented, and diabetic patients were de facto classified as grade $\mathrm{C}$. Moreover, the authors did not record some information like $\mathrm{Pl}$, mastication, and occlusion disorders. This situation suggests some bias in the classification. Since the difference between stage III and IV is mainly based on the complexity factors ${ }^{1,6,16}$. However, the fact that our study was conducted in a university setting, a reference care centre for the treatment of periodontal disease, may in part explain why patients showing up for consultation are generally at an advanced stage of the disease. The poverty level (46.7\%) and belonging to the informal sector characterised by low daily incomes may also justify the delay in consultation and treatment. Periodontal treatment can be estimated at around US\$69 in our context where the minimum income is US\$106.36. ${ }^{17,18}$

A significant relation was observed between the severity of the damage, the BOP and tooth loss experience. Fear of causing 


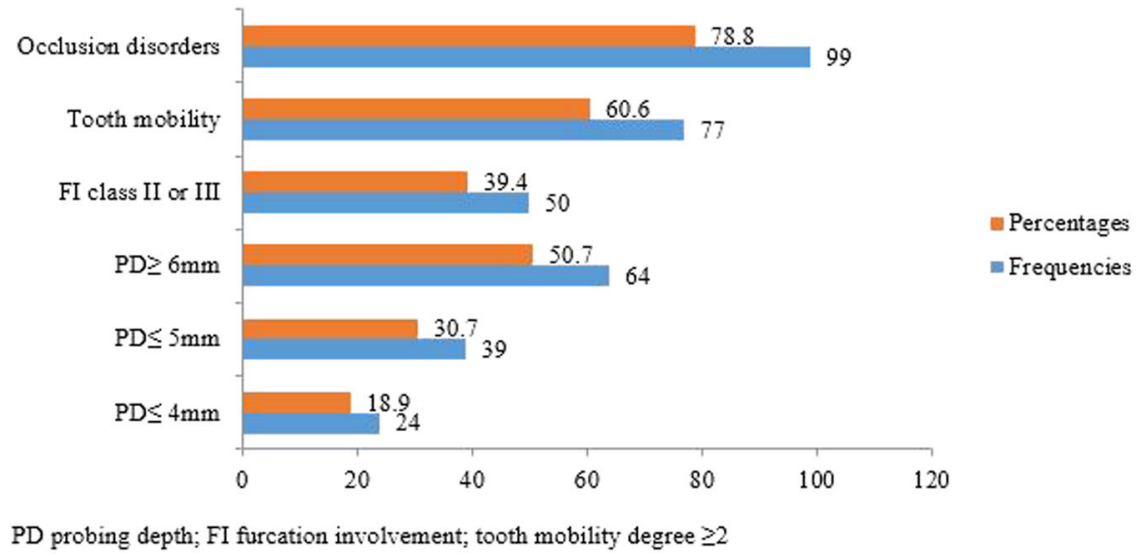

Fig. 3 Clinical features. Showing main clinical characteristics distribution of the study population.

\begin{tabular}{|llr|}
\hline Table 5. Radiographic characteristics. & & \\
\hline Variables & $N=127$ & $\%$ \\
\hline Bone loss/age & & \\
$\quad<0.25$ & 4 & 3.1 \\
0.25 à 1.0 & 39 & 30.7 \\
$>1.0$ & 84 & 66.1 \\
Phenotype & & \\
$\quad$ Heavy biofilm/less destruction & 33 & 26.0 \\
$\quad$ Proportional & 78 & 61.4 \\
$\quad$ Light biofilm/ a lot of destruction & 16 & 12.6 \\
Bone loss extension & & \\
$\quad$ Generalized & 104 & 81.9 \\
$\quad$ Localised & 23 & 18.1 \\
Alveolysis type & & \\
$\quad$ Horizontal & 36 & 28.3 \\
$\quad$ Vertical & 7 & 5.5 \\
$\quad$ Mixed & 84 & 66.1 \\
General bone loss & & \\
$\quad$ Superficial (coronal third) & 12 & 9.4 \\
Moderate (middle third) & 69 & 54.3 \\
Terminal (apical third) & 46 & 36.2 \\
Ridge defect & & \\
$\quad$ Mild (<33\%) & 97 & \\
Moderate (33-50\%) & 51 & \\
Severe (>50\%) & & \\
\hline
\end{tabular}

bleeding or of further mobilizing already loose teeth during daily tooth brushing promotes deterioration of periodontal status and may explain this relation.

However, the study findings are to be interpreted within the confines of certain limitations and comments. Periodontal charting was carried out by students though crosschecked by a periodontal specialist. The periodontal aetiology of tooth loss was not verified due to the memory bias of patients who reported it. ${ }^{14}$ The number of missing teeth in this study was not discriminated against based on origin, which could lead to misclassification. Moreover, wisdom teeth were included in the missing teeth's count during data analysis. The lack of consensus regarding the assessment of radiographic parameters in the literature has led to methodological choices that may be questionable: assessment of alveolysis, characterisation of ridge defect and vertical bone loss

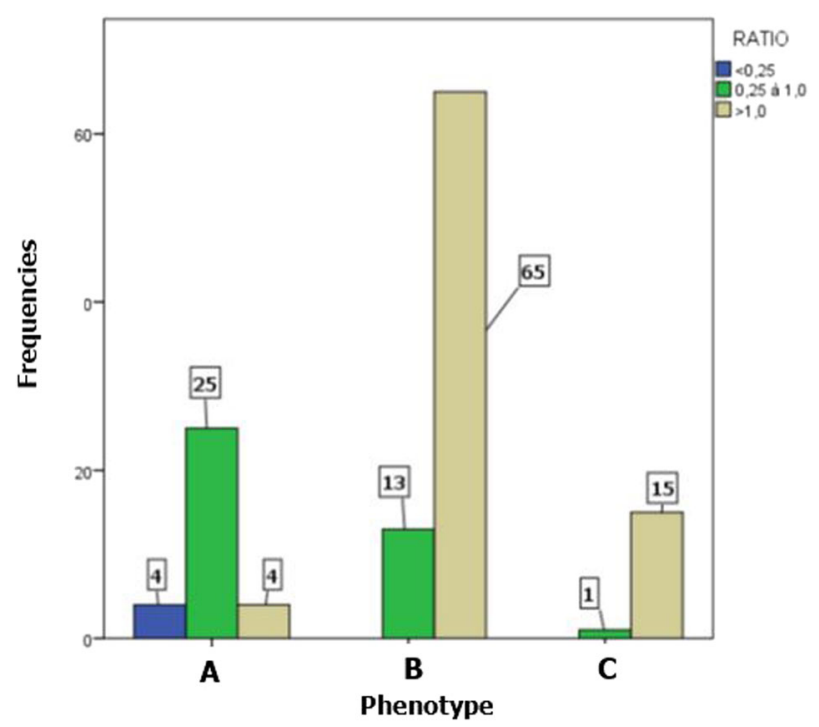

Fig. 4 Ratio of bone loss percentage to age and phenotype. Showing a discrepancy, according to phenotype, grade B periodontitis patients have mostly a ratio greater than one.

Table 6. Distribution of periodontitis according to the stage, grade and extension.

\begin{tabular}{llr}
\hline Variables & $N=127$ & $\%$ \\
\hline Stage & & \\
I & 2 & 1.6 \\
II & 6 & 4.7 \\
III & 31 & 24.4 \\
IV & 89 & 70.1 \\
Grade & & \\
A & 5 & 3.9 \\
B & 34 & 26.8 \\
C & 88 & 69.3 \\
Extent and distribution & & 81.9 \\
Generalized & 104 & 16.5 \\
Localised & 21 & 1.6 \\
Molar/incisor pattern & 2 & \\
\hline
\end{tabular}




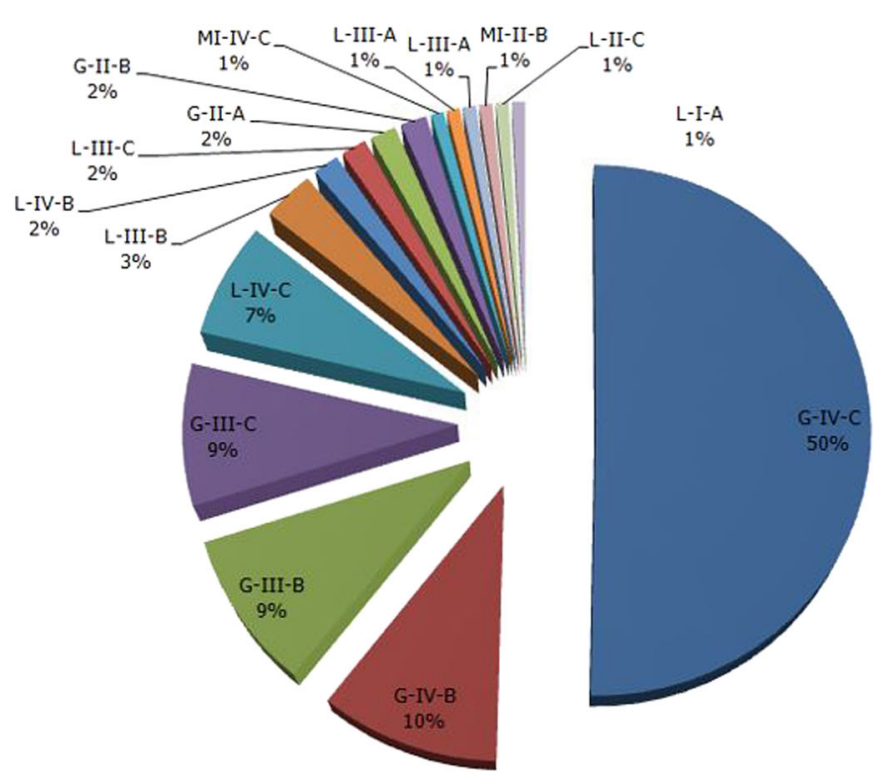

G Generalised; L localised; MI Molar/Incisor; I stage I; II stage II; III stage III; IV stage IV; A grade A; B grade B; C grade C.

Fig. 5 Periodontitis distribution according to clinical forms. Showing generalised stade IV grade $C$ periodontitis as the main clinical form encountered.

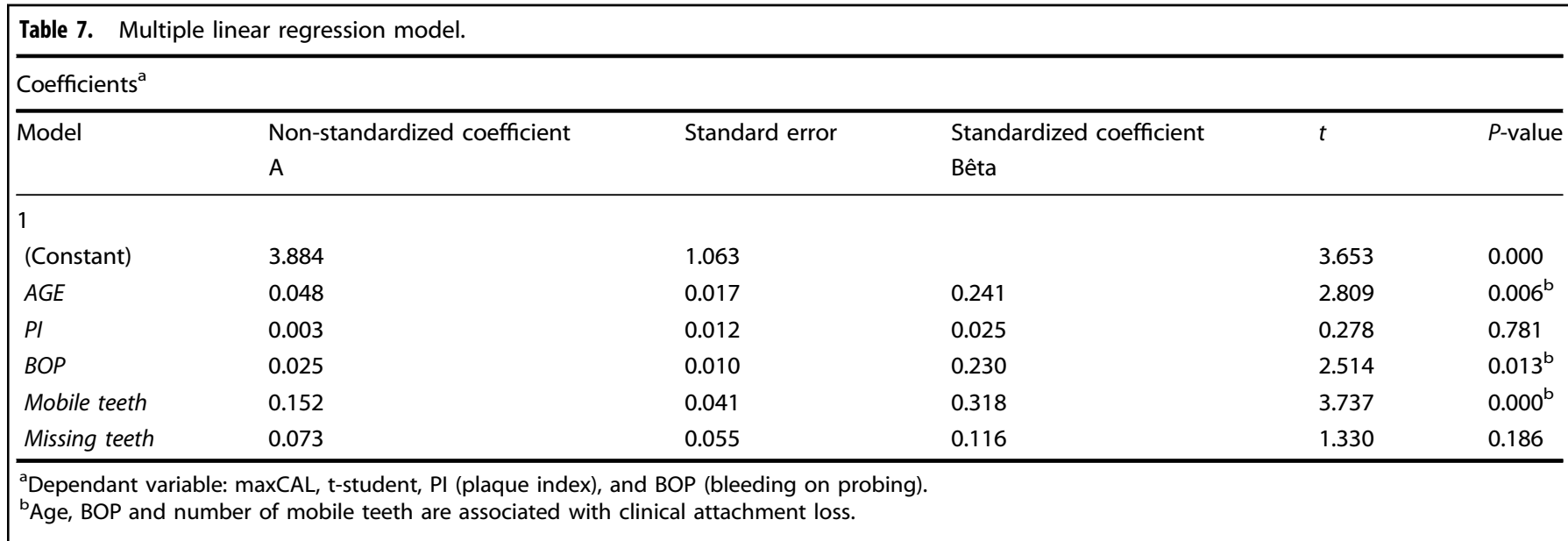

evaluation. Intra-examiner reproducibility has not been determined (intra-class correlation coefficient). Kormann's method for assessing bone lysis leaves some grey zones in subjectivity when it comes to converting this bone loss into a percentage. ${ }^{11}$ Concerning severity criteria for the stage diagnosis, bone loss was not considered as a criterion, the analysis is based mainly on the maximum interdental CAL as advocated by the new classification. $^{6,16}$ Indeed, the use of bone loss percentage is to be considered for staging in the absence of data on CAL.,11 Furthermore, the most clinically affected tooth is not always the most affected on radiographic analysis, thus motivating the choice of maxCAL as the basis for diagnosis.

In the diagnosis relating to grade, the absence of a periodontal history of patients coming for consultation and the noncomputerisation of medical records made it difficult to consider direct evolutionary criteria such as loss of clinical attachment or bone loss over the last 5 years. ${ }^{6}$ Risk factors were documented in this study. The dissociation between phenotype and percentage of bone loss raises the need for objectivity in the characterisation of the patients' phenotype. Indeed, dental biofilm is susceptible to be removed and go to self-reconstitution, while bone loss is irreversible without therapy. The ratio of bone loss percentage to age is the main criterion for determining grade in the absence of periodontal history. ${ }^{16}$

\section{CONCLUSION}

This NCPD brings out a new perspective on patients' periodontal status. Periodontitis, through its characterization according to stage and grade, sees its extent and complexity considered. Periodontitis patients in this study had advanced forms of the disease with end-stage clinical features and extensive radiologic bone destruction. Clinical hindsight is necessary to adapt this classification to practice in limited-resource healthcare settings as it is in developing countries.

\section{AUTHOR CONTRIBUTIONS}

W.N.B., D.T., N.L.B., and A.S. came up with the idea. W.N.B., D.T., N.L.B., A.S., M.L.G., and A.M.D. designed the protocol. W.N.B., D.T., A.S., N.L.B. contributed to data acquisition, synthesis, visualisation, and interpretation. W.N.B., D.T. wrote up the manuscript. M.L.G., A.M.D., A.D., A.S.D., and H.M.B. contributed to data visualisation and 
interpretation, provision of and management of study literature resources. All the authors critically revised the final paper and approved changes prior to publication.

\section{ADDITIONAL INFORMATION}

Competing interests: The authors declare no competing interests.

Publisher's note Springer Nature remains neutral with regard to jurisdictional claims in published maps and institutional affiliations.

\section{REFERENCES}

1. Papapanou, P. N. et al. Periodontitis: consensus report of workgroup 2 of the 2017 World Workshop on the classification of periodontal and peri-implant diseases and conditions: classification and case definitions for periodontitis. J. Clin. Periodontol. 45, S162-S170 (2018).

2. Pihlstrom, B. L., Michalowicz, B. S. \& Johnson, N. W. Periodontal diseases. Lancet 366, 1809-1820 (2005).

3. Armitage, G. C. Development of a classification system for periodontal diseases and conditions. Ann. Periodontol. 4, 1-6 (1999).

4. Caton, J. G. et al. A new classification scheme for periodontal and peri-implant diseases and conditions - Introduction and key changes from the 1999 classification. J. Clin. Periodontol. 45, S1-S8 (2018).

5. Page, R. C. \& Schroeder H. E. Periodontitis in Man and Other Animals. 5-57 (Karger, Basel, 1982).

6. Tonetti, M. S., Greenwell, H. \& Kornman, K. S. Staging and grading of periodontitis: framework and proposal of a new classification and case definition. J. Clin. Periodontol. 45, S149-S161 (2018).

7. Mühlemann, H. R. Tooth mobility: the measuring method. initial and secondary tooth mobility. J. Periodontol. 25, 22-29 (1954).

8. O'Leary, T. J., Drake, R. B. \& Naylor, J. E. The plaque control record. J. Periodontol. 43, 38-40 (1972).

9. Ainamo, J. \& Bay, I. Problems and proposals for recording gingivitis and plaque. Int Dent. J. 25, 229-235 (1975).

10. Hamp, S.-E., Nyman, S. \& Lindhe, J. Periodontal treatment of multi rooted teeth. J. Clin. Periodontol. 2, 126-135 (1975).

11. Kornman, K. S. \& Papapanou, P. N. Clinical application of the new classification of periodontal diseases: Ground rules, clarifications and "gray zones". J. Periodontol. 91, 352-360 (2020)
New classification of periodontal diseases (NCPD): an application in a... W Ndjidda Bakari et al.

12. Benoist, H. M. et al. Profile of chronic and aggressive periodontitis among Senegalese. J. Periodontal Implant Sci. 41, 279-284 (2011).

13. Ravidà, A. et al. Using periodontal staging and grading system as a prognostic factor for future tooth loss: a long-term retrospective study. J. Periodontol. 91, 454-461 (2020).

14. Graetz, C. et al. Comparison of periodontitis patients' classification in the 2018 versus 1999 classification. J. Clin. Periodontol. 46, 908-917 (2019).

15. Papapanou, P.N. \& Lindhe J. Epidemiology of periodontal diseases. In Clinical Periodontology and Implant Dentistry 6th edn (eds Lang, N. P. \& Lindhe, J.) 125-156 (John Wiley \& Sons, London, 2015).

16. Tonetti, M. S. \& Sanz, M. Implementation of the new classification of periodontal diseases: decision-making algorithms for clinical practice and education. J. Clin. Periodontol. 46, 398-405 (2019).

17. National Agency for Statistics and Demography. Communication of the Poverty Results of the Harmonised Survey on Household Living Conditions in Senegal (2020). http://www.ansd.sn/index.php?option=com_content\&view=article\&id=635:202007-23-09-31-04\&catid=56:depeches\&ltemid=264 Accessed Nov 2020.

18. JeuneAfriqueEconomie \& finance. Senegal: Minimum Income Increased for the First Time Since 1996 (2018) https://www.jeuneafrique.com/556925/economie/senegal-le-salaire-minimum-revalorise-pour-la-premiere-fois-depuis-1996/ Accessed Nov 2020.

(i) Open Access This article is licensed under a Creative Commons Attribution 4.0 International License, which permits use, sharing, adaptation, distribution and reproduction in any medium or format, as long as you give appropriate credit to the original author(s) and the source, provide a link to the Creative Commons license, and indicate if changes were made. The images or other third party material in this article are included in the article's Creative Commons license, unless indicated otherwise in a credit line to the material. If material is not included in the article's Creative Commons license and your intended use is not permitted by statutory regulation or exceeds the permitted use, you will need to obtain permission directly from the copyright holder. To view a copy of this license, visit http://creativecommons. org/licenses/by/4.0/.

(c) The Author(s) 2021 